\title{
Phase-only laser control in the weak-field limit: Two-pulse control of IBr photofragmentation revisited
}

Tiwari, Ashwani Kumar; Henriksen, Niels Engholm

Published in:

Journal of Chemical Physics

Link to article, DOI:

$10.1063 / 1.4939247$

Publication date:

2016

Document Version

Publisher's PDF, also known as Version of record

Link back to DTU Orbit

Citation (APA):

Tiwari, A. K., \& Henriksen, N. E. (2016). Phase-only laser control in the weak-field limit: Two-pulse control of IBr photofragmentation revisited. Journal of Chemical Physics, 144(1), [014306]. https://doi.org/10.1063/1.4939247

\section{General rights}

Copyright and moral rights for the publications made accessible in the public portal are retained by the authors and/or other copyright owners and it is a condition of accessing publications that users recognise and abide by the legal requirements associated with these rights.

- Users may download and print one copy of any publication from the public portal for the purpose of private study or research.

- You may not further distribute the material or use it for any profit-making activity or commercial gain

- You may freely distribute the URL identifying the publication in the public portal 


\section{A|P| $\mid \begin{aligned} & \text { The Journal of } \\ & \text { Chemical Physics }\end{aligned}$}

Phase-only laser control in the weak-field limit: Two-pulse control of IBr photofragmentation revisited

Ashwani K. Tiwari and Niels E. Henriksen

Citation: The Journal of Chemical Physics 144, 014306 (2016); doi: 10.1063/1.4939247

View online: http://dx.doi.org/10.1063/1.4939247

View Table of Contents: http://scitation.aip.org/content/aip/journal/jcp/144/1 ?ver=pdfcov

Published by the AIP Publishing

\section{Articles you may be interested in}

Phase-only shaped laser pulses in optimal control theory: Application to indirect photofragmentation dynamics

in the weak-field limit

J. Chem. Phys. 136, 044303 (2012); 10.1063/1.3678013

Coherent control of indirect photofragmentation in the weak-field limit: Control of transient fragment distributions

J. Chem. Phys. 134, 164308 (2011); 10.1063/1.3582928

Quantum control of molecular orientation by two-color laser fields

J. Chem. Phys. 120, 5176 (2004); 10.1063/1.1644102

Two-pulse laser control for selective photofragment orientation

J. Chem. Phys. 111, 3051 (1999); 10.1063/1.479585

Quantum control of dynamics

AIP Conf. Proc. 388, 69 (1997); 10.1063/1.52153

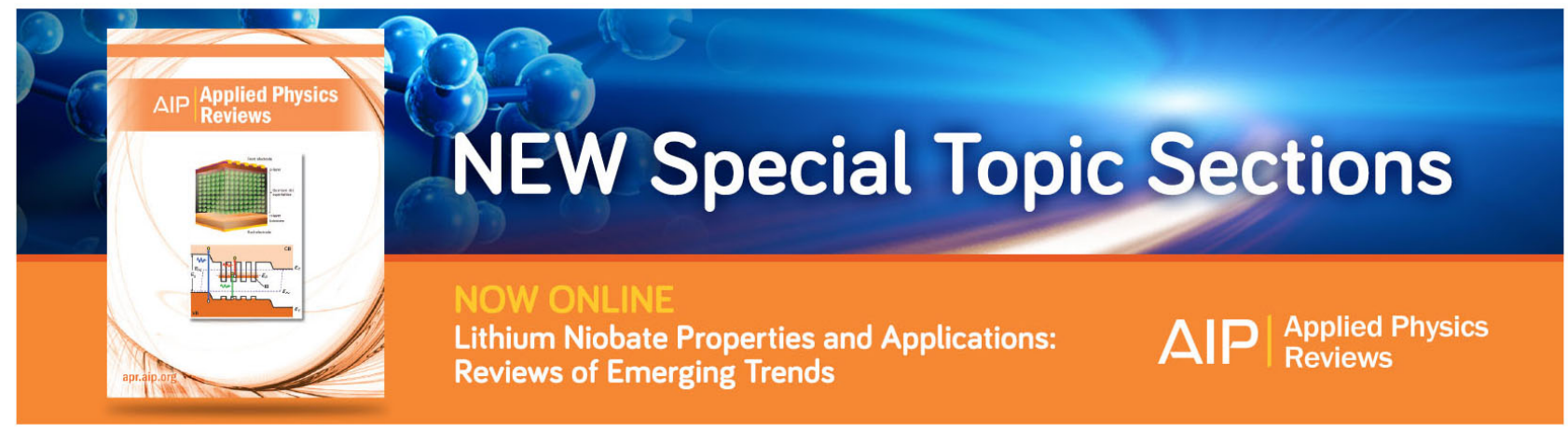




\title{
Phase-only laser control in the weak-field limit: Two-pulse control of IBr photofragmentation revisited
}

\author{
Ashwani K. Tiwari ${ }^{1, a)}$ and Niels E. Henriksen ${ }^{2, b)}$ \\ ${ }^{1}$ Indian Institute of Science Education and Research Kolkata, Mohanpur 741 246, India \\ ${ }^{2}$ Department of Chemistry, Technical University of Denmark, Building 207, DK-2800 Kongens Lyngby, \\ Denmark
}

(Received 13 November 2015; accepted 18 December 2015; published online 6 January 2016)

\begin{abstract}
We demonstrate theoretically that laser-induced coherent quantum interference control of asymptotic states of dissociating molecules is possible, starting from a single vibrational eigenstate, after the interaction with two laser pulses - at a fixed time delay-both operating in the weak-field limit. Thus, phase dependence in the interaction with the second fixed-energy phase-modulated pulse persists after the pulse is over. This is illustrated for the nonadiabatic process: $\mathrm{I}+\mathrm{Br}^{*}$ $\leftarrow \mathrm{IBr} \rightarrow \mathrm{I}+\mathrm{Br}$, where the relative yield of excited $\mathrm{Br}^{*}$ can be changed by pure phase modulation. Furthermore, a strong frequency dependence of the branching ratio is observed and related to the re-crossing dynamics of the avoided crossing in the above-mentioned process. ( 2016 AIP Publishing LLC. [http://dx.doi.org/10.1063/1.4939247]
\end{abstract}

\section{INTRODUCTION}

Laser control of matter takes advantage of the phase coherence as well as intensity of laser light. ${ }^{1-11}$ The frequency components of a laser pulse can be represented as $A(\omega) \exp [i \phi(\omega)]$, i.e., as an amplitude and a phase $\phi(\omega)$. Control via pure phase-modulation of laser light has been demonstrated in numerous experimental studies (see, e.g., Refs. 4-7). The experimental studies have with a few exceptions all been conducted in the strong-field limit.

In the weak-field (one-photon) limit for gas-phase photofragmentation of isolated molecules with the system initially in an eigenstate of the ground electronic state, it has been shown that in the long-time limit, all observables associated with the fragments are independent of the spectral phases of the laser light. ${ }^{12-15}$ That is, for this particular scenario, the phase coherence of laser light is not playing any role. We note in passing that under different circumstances, weak-field phase control might be possible in the long-time limit, e.g., for isomerization and open quantum systems. ${ }^{14}$

Although no phase-modulation is possible in the longtime limit where dissociation is completed, we have demonstrated that phase-modulation of the outcome of "early" dynamics is possible. Thus, transient post-pulse phase effects on the total dissociation probability ${ }^{16-18}$ and electronic branching ratio $^{18}$ have been predicted. Furthermore, pure phase-modulation control of the final vibrational states in the dissociation of the van der Waals complex $\mathrm{Ne}-\mathrm{Br}_{2}$ $\rightarrow \mathrm{Ne}+\mathrm{Br}_{2}$ was predicted, ${ }^{15}$ and it was shown that this control of the distribution of relative translational energy to internal vibrational energy was possible due to the presence of overlapping resonances. The transient effects were shown to be particularly "long-lived" for indirect dissociation ${ }^{15-17}$ where

\footnotetext{
a)Electronic address: ashwani@iiserkol.ac.in

b) Electronic address: neh@kemi.dtu.dk
}

an intermediate energized complex is formed, i.e., products may then continuously "leak out" of the complex over an extended period of time and a phase dependence on the "early" products can be observed. These phase effects can be observed by time-resolved detection and, in principle, be isolated from the ensuing dynamics via a time-resolved spatial separation technique, e.g., using time-dependent alignment of the photo-excited molecules.

In the present work, we stay within the weak-field limit but allow now for two sequential one-photon excitations. This is similar to many pump-probe schemes for molecular dynamics, here with the probe pulse replaced by a control pulse. Weak-field excitation is attractive because unwanted processes like ionization are avoided.

In this context, it is well-known that the outcome can depend on the time delay between the two pulses. A time shift of a laser pulse is introduced by a linear spectral phase function, i.e., $\phi(\omega)=\alpha\left(\omega-\omega_{0}\right)$, where $\alpha$ is a constant. It has even been shown in model studies that the carrier envelope phase, $\phi(\omega)=\phi_{0}$, of the second (multi-cycle) pulse can play a role. ${ }^{19,20}$ Since the first pulse can create a nonstationary superposition of eigenstates, the original seminal work of Brumer and Shapiro ${ }^{21}$ suggests that a general phase modulation of the second pulse will allow for the manipulation of quantum mechanical interference terms.

Thus, assume that a nonstationary state has been created in an electronic state

$$
\left|\chi_{1}(t)\right\rangle=\sum_{i} c_{i} e^{-i \epsilon_{i} t / \hbar}|i\rangle
$$

where $|i\rangle$ and $\epsilon_{i}$ are the nuclear (vibrational-rotational) eigenstates and associated eigenvalues, which are here assumed to be discrete. The nuclear wave packet created in a subsequent electronic transition, described within first-order perturbation theory, can then (see Ref. 22 for details) be 
written in the form

$$
\left|\chi_{2}(t)\right\rangle=\frac{i}{\hbar} \sum_{i} \int_{0}^{t} d t^{\prime} e^{-i \epsilon_{i} t^{\prime} / \hbar} \mathcal{E}\left(t^{\prime}\right) e^{-i H\left(t-t^{\prime}\right) / \hbar}\left|\phi_{i}\right\rangle,
$$

where $\mathcal{E}(t)$ is the electric field associated with the laser excitation, $H$ is the nuclear Hamiltonian of the excited electronic state, and $\left|\phi_{i}\right\rangle=c_{i} \boldsymbol{\mu}_{21} \cdot \boldsymbol{e}_{0}|i\rangle$, where $\boldsymbol{\mu}_{21}$ is the transition-dipole moment vector of the electronic transition and $\boldsymbol{e}_{0}$ is a unit polarization vector of the plane-polarized field.

We write the time-dependent phase-coherent laser field in the form

$$
\mathcal{E}(t)=\mathcal{E}_{0} \operatorname{Re}\left[\int_{-\infty}^{\infty} A(\omega) e^{i \phi(\omega)} e^{-i \omega t} d \omega\right],
$$

where $A(\omega)$ is the real-valued distribution of frequencies and $\phi(\omega)$ is the real-valued frequency-dependent phases, and consider a photofragmentation process, i.e., $\mathrm{ABC} \rightarrow \mathrm{A}+\mathrm{BC}$. The probability of observing the fragments in an eigenstate $|E, n\rangle$ with quantum number(s) $n$, at the total energy $E$, after the excitation pulse $\mathcal{E}(t)$ has vanished and in the long-time limit where dissociation is completed, ${ }^{22}$ can be written in the form

$$
P(E, n)=C\left|\sum_{i} A\left(\omega_{i}\right) e^{i \phi\left(\omega_{i}\right)} p_{i}(E, n)\right|^{2},
$$

where $p_{i}(E, n)=\lim _{t \rightarrow \infty}\left\langle E, n|\exp (-i H t / \hbar)| \phi_{i}\right\rangle$ is related to the dissociation amplitude out of the eigenstate $|i\rangle, \omega_{i}$ $=\left(E-\epsilon_{i}\right) / \hbar$, and $C$ is a constant.

Equation (4) is a generalization of an expression which has been presented previously (see Ref. 15) and it shows clearly that (i) for excitation out of a single eigenstate, the dependence on the laser phases is absent, and (ii) for excitation out of a sum of eigenstates, pure phase modulation can change the quantum mechanical (off-diagonal) interference terms in the probability. Note that this expression treats all forms of phase modulation on equal footing. Thus, the introduction of a linear spectral phase function $\phi(\omega)=\alpha\left(\omega-\omega_{0}\right)$ equivalent to the time shift $\mathcal{E}(t) \rightarrow \mathcal{E}(t-\alpha)$ of the laser pulse in Eq. (3) is just one particular simple example of a phase modulation.

\section{TWO-PULSE EXCITATION OF IBr}

In order to investigate the possibilities of phaseonly control suggested by Eq. (4), we consider the photofragmentation of the $\mathrm{IBr}$ molecule leading to atomic fragments in two different electronic states

$$
\mathrm{IBr}+\text { coherent light } \longrightarrow\left\{\begin{array}{l}
\mathrm{I}+\mathrm{Br}^{*} \\
\mathrm{I}+\mathrm{Br}
\end{array},\right.
$$

where $\mathrm{Br}^{*}$ is the spin-orbit excited $\operatorname{Br}\left({ }^{2} \mathrm{P}_{1 / 2}\right)$. There are several previous works related to this photo-induced process, theoretical (see, e.g., Refs. 18, 23, and 24) as well as experimental (see, e.g., Refs. 9 and 25).

The relevant potential energy curves for $\mathrm{IBr}^{24,25}$ are shown in Fig. 1. We consider in the following laser excitation with $\mathrm{IBr}$ initially in the vibrational ground state of the electronic ground state $(X)$. A bound excited state $(A)$ and two higher excited state potentials ( $B$ and $Y$ ) which interact with each

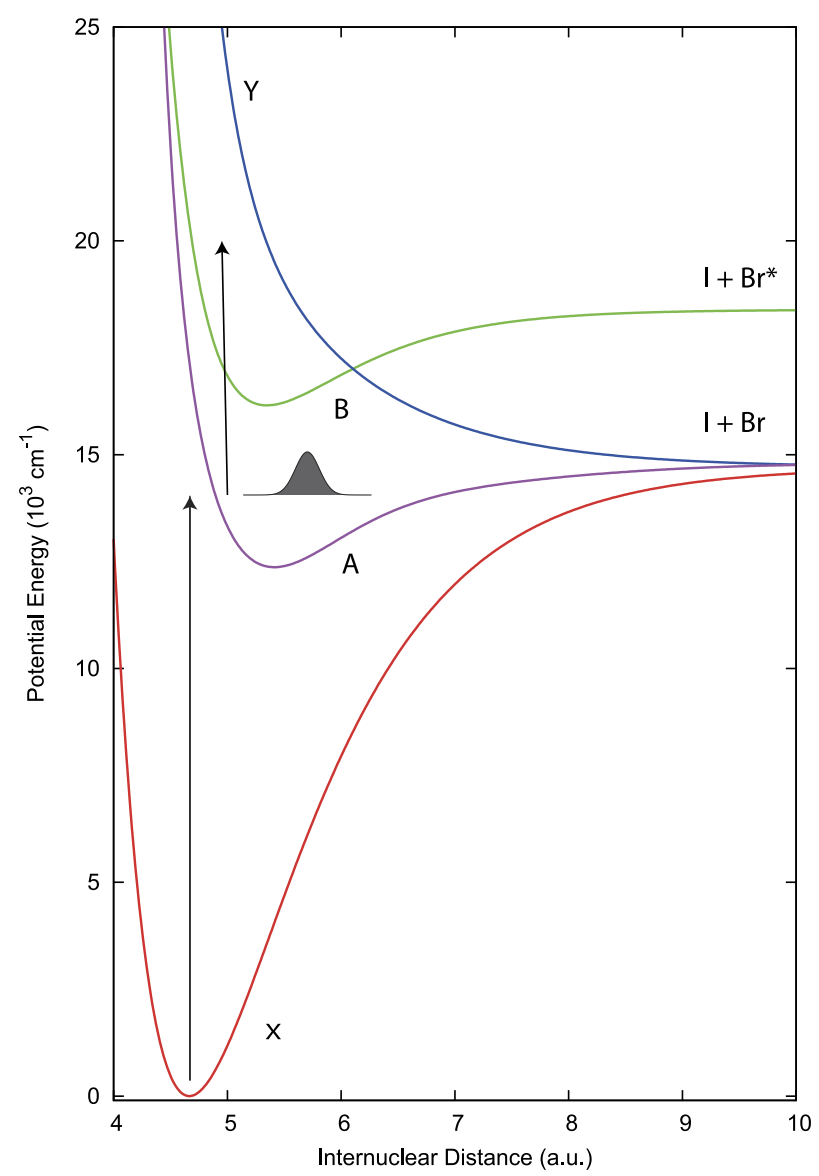

FIG. 1. The relevant potential energy curves of $\mathrm{IBr}$ and a sketch of the two-step excitation $X \rightarrow A \rightarrow B$. The wave packet created in the bound $A$ state is shown. Due to the crossing between the $B$ and $Y$ states, the wave packet created in the $A \rightarrow B$ transition bifurcates into the two channels $\mathrm{I}+\mathrm{Br}^{*}$ and $\mathrm{I}+\mathrm{Br}$.

other around their crossing at an internuclear distance of about 6 a.u. are included. The $A$-state potential is extracted from spectroscopic observations ${ }^{25}$ and the $B$ and $Y$ potentials including the diabatic coupling potential ${ }^{24}$ are known to reproduce the experimentally observed branching ratio $\mathrm{Br}^{*} / \mathrm{Br}$ at low energies (i.e., $500 \leq \lambda<545 \mathrm{~nm}$ ) in the $X \rightarrow B$ transition. Additional excited electronic states are involved at higher energies. ${ }^{25}$ Excitation for $\lambda<545 \mathrm{~nm}\left(18349 \mathrm{~cm}^{-1}\right)$ is above the dissociation limit for both channels.

It is noted that a two-pulse control scheme has been suggested in this context. ${ }^{23}$ However, only the time delay was varied, that is, (nonlinear) optical phases were not included. Furthermore, the nonstationary wave packet in the $A$ state was assumed to consist of a superposition of only 2 bound states, and the second pulse promoted this wave packet into the $Y$ state (with an excitation energy around $15000 \mathrm{~cm}^{-1}$ ).

In the following, we employ two non-overlapping Gaussian laser pulses. The first pulse $\left(\tau_{\mathrm{FWHM}}=100 \mathrm{fs}\right)$ creates a wave packet in the $A$ state and, at a fixed time delay, a second Gaussian pulse with fixed bandwidth and different linear temporal chirps $\left(\tau_{\mathrm{FWHM}}=10 \mathrm{fs}\right.$ for the unchirped pulse $)$ promotes the wave packet from the $A$ to the $B$ state (again the time delay corresponds to a given linear spectral phase of the second pulse). 
The Gaussian frequency distribution (centered at $\omega_{i}$ ) including a quadratic phase function is given by

$$
A(\omega) e^{i \phi(\omega)}=\sqrt{\frac{\tau_{0}^{2}}{2 \pi}} e^{\left[-\tau_{0}^{2}\left(\omega-\omega_{i}\right)^{2} / 2+i \beta_{0}\left(\omega-\omega_{i}\right)^{2} / 2\right]},
$$

where $1 / \tau_{0}$ is the frequency bandwidth and $\beta_{0}$ is the quadratic spectral chirp. The time-dependent phase-coherent electric field of the laser pulse becomes

$$
\begin{aligned}
\mathcal{E}(t) & =\mathcal{E}_{0} \operatorname{Re}\left[\int_{-\infty}^{\infty} A(\omega) e^{i \phi(\omega)} e^{-i \omega t} d \omega\right] \\
& =\mathcal{E}_{0} \operatorname{Re}\left[\sqrt{\frac{\tau_{0}^{2}}{\tau_{0}^{2}-i \beta_{0}}} \exp \left(-\frac{t^{2}}{2 \tau^{2}}-i \beta t^{2} / 2-i \omega_{i} t\right)\right]
\end{aligned}
$$

with pulse duration $\tau$ and linear temporal chirp $\beta$, which are related to $\tau_{0}$ and $\beta_{0}$ via $\tau^{2}=\tau_{0}^{2}\left(1+\beta_{0}^{2} / \tau_{0}^{4}\right)$ and $\beta$ $=\beta_{0} /\left(\tau_{0}^{4}+\beta_{0}^{2}\right)$. For $\beta_{0}=0, \beta=0$ and $\tau=\tau_{0}$ and the result is identical to the transform-limited Gaussian pulse used in the first excitation step.

The spectral chirp introduces a time-dependent frequency distribution of the field in Eq. (7). For the second pulse, we start with a transform-limited pulse $\left(\beta_{0}=\beta=0\right)$ of duration $\tau_{\mathrm{FWHM}}=\tau \sqrt{8 \ln 2}=10 \mathrm{fs}$, keep the bandwidth $1 / \tau_{0}$ fixed, and change then the quadratic spectral chirp $\beta_{0}$. We consider laser pulses with a maximum chirp corresponding to $\beta_{0}=1000$ and $-1000 \mathrm{fs}^{2}$. With this chirp, the temporal duration of the Gaussian pulse envelope is still within a few hundred femtoseconds, i.e., $\tau_{\mathrm{FWHM}}=235.5 \mathrm{fs}$.

The sum of the center frequencies $v_{1}+v_{2}$ (in the following reported as wavenumbers) of the two pulses is fixed at $20353 \mathrm{~cm}^{-1}$ which is close to $500 \mathrm{~nm}$. We choose $v_{1}=14384 \mathrm{~cm}^{-1}\left(\lambda_{1}=695.2 \mathrm{~nm}\right)$ and $v_{2}=5969 \mathrm{~cm}^{-1}$ $\left(\lambda_{1}=1780.6 \mathrm{~nm}\right)$ and include the possibility of frequency control at a fixed total energy by changing $\Delta v$ in $v_{1}+\Delta v$ and $v_{2}-\Delta v$ by $\pm 200 \mathrm{~cm}^{-1}$.

We treat $\mathrm{IBr}$ as a one-dimensional system (see Ref. 24 for details), assuming that the transition dipole moments for the two electronic transitions are oriented in the direction of the fields. The laser-induced dynamics are calculated within the electric-dipole approximation and first-order perturbation theory for the interaction with each pulse. The wave functions, potentials, and coupling element are represented on an equally spaced grid of 1024 points in the range $3.70 \leq x \leq 20.50$ a.u. An absorbing potential is added for $x \geq 18.4$ a.u. to avoid unphysical reflections into the inner region. The initial wave function, the vibrational ground state of the $X$ state, is computed using the Fourier-grid Hamiltonian method. ${ }^{26}$ In the diabatic representation, the time propagation of wave functions, $\psi_{i}(x, t)$, where $i$ refers to channel $\mathrm{I}+\mathrm{Br}^{*}$ or $\mathrm{I}+\mathrm{Br}$, is accomplished by the split-operator method. ${ }^{27}$ We propagate the wave function for a total time of $120 \mathrm{ps}$ since the dissociation dynamics can be very slow for some of the above specified frequencies.

The intensity of each pulse is $1.7 \times 10^{10} \mathrm{~W} / \mathrm{cm}^{2}$ (with a constant transition dipole moment of 1 a.u.), such that the calculations are performed in the weak-field regime where the excitation probability depends linearly on laser intensity.
The excitation probability for each pulse is less than $1 \%$, depending on the choice of $\Delta v$.

We consider the normalized/relative yield of $\mathrm{Br}^{*}$ in the two channels $\mathrm{I}+\mathrm{Br}^{*}$ and $\mathrm{I}+\mathrm{Br}$, defined as the converged ratio $P_{\mathrm{Br}^{*}} /\left(P_{\mathrm{Br}^{*}}+P_{\mathrm{Br}}\right)$ in the long-time limit. The dissociation probability

$$
P_{i}(t)=\int_{-\infty}^{t} J_{i}\left(t^{\prime}\right) d t^{\prime}
$$

is obtained as the time-integrated flux over $[-\infty, t]$, where the probability flux at $x=x_{d}$ is given by

$$
J_{i}(t)=\frac{\hbar}{m} \operatorname{Im}\left[\psi_{i}^{*}(x, t) \frac{\partial \psi_{i}(x, t)}{\partial x}\right]_{x=x_{d}},
$$

where $m$ is the reduced mass of IBr. In the following, we choose $x_{d}=10$ a.u. which is within $\sim 150 \mathrm{~cm}^{-1}$ from the asymptotic value of the potentials.

\section{RESULTS AND DISCUSSION}

First, we consider the dissociation dynamics employing laser pulses without any chirp, i.e., $\beta_{0}=0$. Figure 2 shows the normalized/relative yield of $\mathrm{Br}^{*}$ as a function of time delay between the two pulses with $\Delta v=0.0$. We observe a quite strong dependence on the time delay.

Figure 3 shows the normalized/relative yield of $\mathrm{Br}^{*}$ as a function of $\Delta v$. The time delay between the two pulses is now fixed at 100 fs. A very strong frequency dependence is observed although the total excitation energy is constant. For reference, the cw result for direct excitation to the $B$ state (out of the $X$ state) gives a branching ratio of 0.72 at $500 \mathrm{~nm}$, with a frequency dependence which is much weaker compared to the two-step excitation.

This frequency dependence is analyzed in Fig. 4 which shows the average momentum as well as the time-integrated flux on the $B$ state for the two extremes $\Delta v=-200 \mathrm{~cm}^{-1}$ and $\Delta v=+200 \mathrm{~cm}^{-1}$ in Fig. 3. The average momentum in the case $\Delta v=+200 \mathrm{~cm}^{-1}$ decreases over time indicating that the wave packet is decelerated by the attractive part of the $B$ state potential. In contrast, for $\Delta v=-200 \mathrm{~cm}^{-1}$, the average

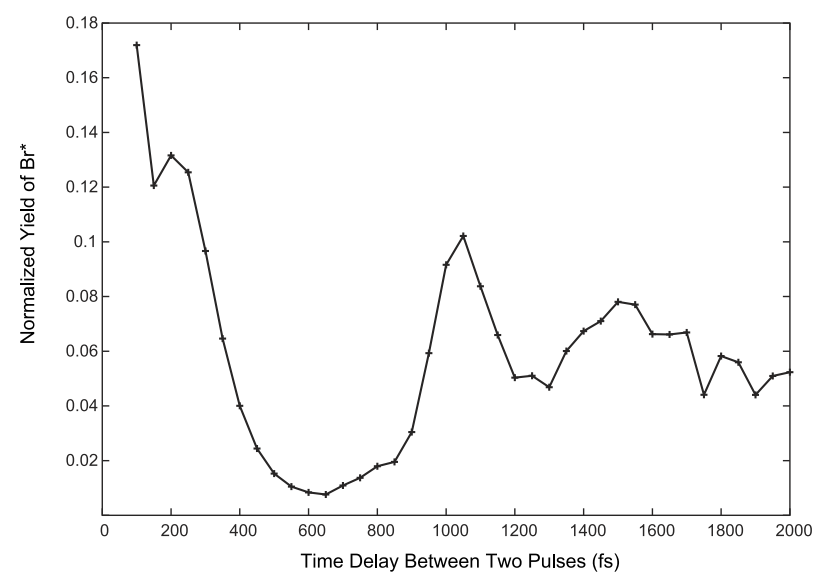

FIG. 2. The normalized/relative yield of $\mathrm{Br}^{*}$ as a function of time delay between the two pulses. $\Delta v=0.0$ and $\beta_{0}=0$ in this case (see text for details). 


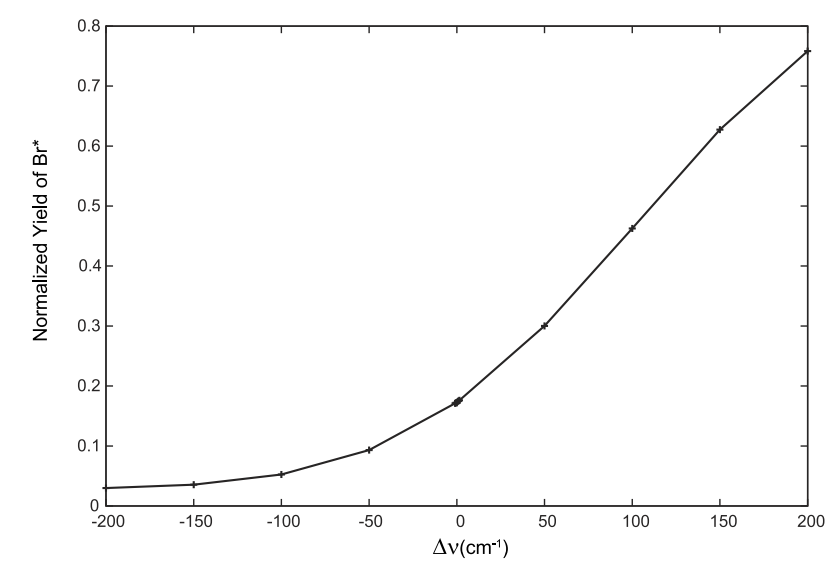

FIG. 3. The normalized/relative yield of $\mathrm{Br}^{*}$ as a function of $\Delta v$. The time delay between the two pulses is $100 \mathrm{fs}$ and $\beta_{0}=0$.

momentum oscillates between positive and negative values indicating a predominantly bound oscillatory motion.

The lower panel shows the corresponding normalized time-integrated flux on the $B$ state as a function of time with the flux line-for the purpose of analyzing the dynamics-at the crossing point between the $B$ and $Y$ states. In the long-time limit, the normalized time-integrated flux on the $B$ state will
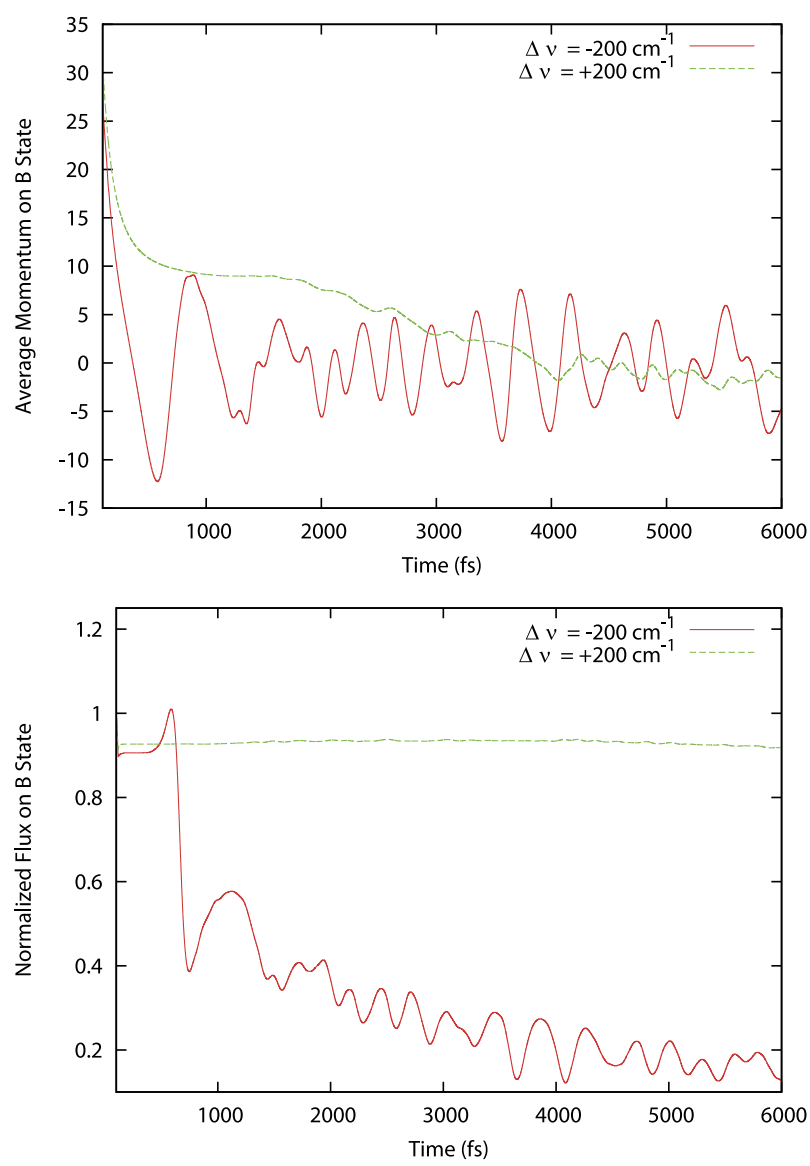

FIG. 4. Upper panel: Average momentum on $B$ state as a function of time. Lower panel: Normalized time-integrated flux on $B$ state as a function of time with the flux line at the crossing point. Note the many recrossings for $\Delta v=-200 \mathrm{~cm}^{-1}$ whereas the dissociation, essentially, is direct for $\Delta v$ $=+200 \mathrm{~cm}^{-1}$. The time delay between the two pulses is $100 \mathrm{fs}$ and $\beta_{0}=0$. approach the normalized yield in Fig. 3. For $\Delta v=+200 \mathrm{~cm}^{-1}$, the time-integrated flux is essentially constant, being the signature of a direct dissociation. The normalized yield is 0.75 according to Fig. 3, thus a small fraction will recombine at long times. In contrast, for $\Delta v=-200 \mathrm{~cm}^{-1}$, the time-integrated flux oscillates, i.e., there are many (partial) recrossings and note that this indirect dissociation is very slow, i.e., the dissociation probability has not converged after $6 \mathrm{ps}$ (converged results are obtained after a total propagation time of $120 \mathrm{ps}$ ).

It is instructive to analyze the branching ratio in terms of the Landau-Zener formalism for curve crossing. For a single crossing, the probability of remaining in the diabatic $B$ state (equivalent to the probability of a non-adiabatic transition) is given by

$$
P_{\mathrm{LZ}}=\exp \left[-2 \pi\left|V_{12}\right|^{2} /\left(\hbar\left|\beta_{2}-\beta_{1}\right| v_{c}\right)\right]
$$

where $V_{12}$ is the (constant) coupling potential between the diabatic states, $\beta_{1}$ and $\beta_{2}$ are the derivatives of the diabatic states at the crossing, and $v_{c}=\langle p\rangle_{c} / m$ is the expectation value of the speed at the time of the crossing.

For the direct $X \rightarrow B$ excitation to the $B$ state, this formula did nicely reproduce the full quantum mechanical results. ${ }^{28}$ In the present case with excitation to the $B$ state via the $A$ state, we observe a much larger variation in the branching ratio, although the total excitation energy is constant. For $\Delta v=+200 \mathrm{~cm}^{-1}$, the exact crossing probability, equivalent to the normalized yield of $\mathrm{Br}^{*}$, is 0.75 . At a time delay of $100 \mathrm{fs}$, the wave packet is promoted to the $B$ state around the crossing region of the $B$ and $Y$ states, i.e., the simple Landau-Zener formula in Eq. (10) should be applied with some caution but we obtain $P_{\mathrm{LZ}}=0.65$ in quite good agreement with the exact result. For $\Delta v=-200 \mathrm{~cm}^{-1}$, we have multiple crossings. The final normalized yield of $\mathrm{Br}^{*}$ is 0.03 . After the first crossing, we obtain $P_{\mathrm{LZ}}=0.61$ according to Eq. (10). If we, e.g., calculate the probability of remaining in the upper adiabatic state with the formation of $\mathrm{Br}^{*}$ after an odd number of crossings, we obtain $0.61\left(1-P_{\mathrm{LZ}}[\leftarrow]\right)\left(1-P_{\mathrm{LZ}}[\rightarrow]\right) \cdots$, where $1-P_{\mathrm{LZ}}[\leftarrow]$ is the probability of remaining in the upper adiabatic state after the second (in going) crossing, etc. Thus, the overall probability will clearly be smaller than 0.61 .

Although the results above show a phase dependence of the second laser pulse in the form of a delay-time dependence, we now turn to a "non-trivial" nonlinear phase-modulation of the second laser pulse. Figure 5 shows the normalized/relative yield of $\mathrm{Br}^{*}$ for different linear temporal chirps of the second pulse. The time delay between the two pulses is now fixed at $1050 \mathrm{fs}$ and $\Delta v=0.0$. The time delay was increased in order to avoid overlap between the two pulses, taking into consideration that introduction of chirp (or any nonlinear phase) increases the temporal duration of the pulse. We observe now genuine coherent control, i.e., phase-only interference control of the branching ratio for excitation at a fixed energy (center frequency and bandwidth are fixed) at a fixed time delay.

A variation in the branching ratio of more than a factor of 5 is demonstrated for a linear temporal chirp. Recall that such a phase dependence is absent for direct excitation of a single eigenstate to the $B$ state. Comparing Fig. 5 with Fig. 2 


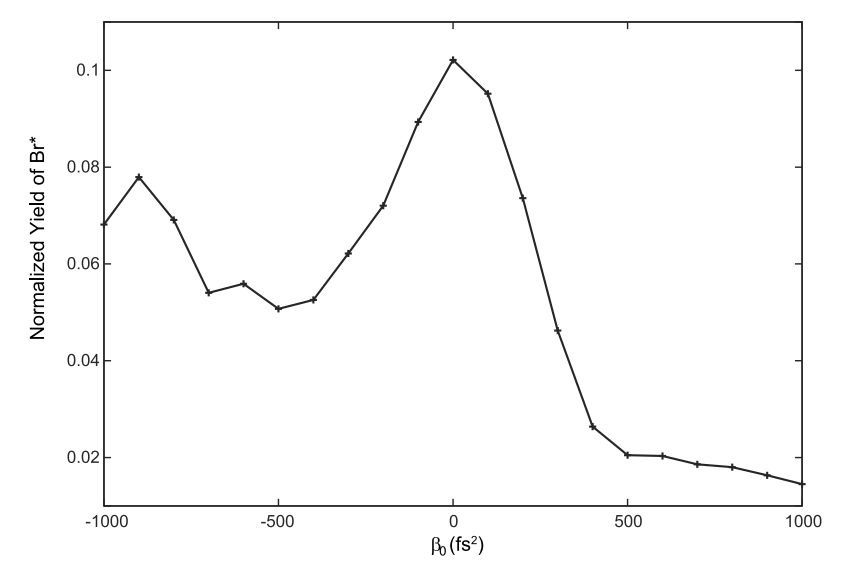

FIG. 5. The normalized/relative yield of $\mathrm{Br}^{*}$ for different quadratic spectral chirps $\beta_{0}$ of the second pulse. The time delay between the two pulses is 1050 fs and $\Delta v=0.0$.

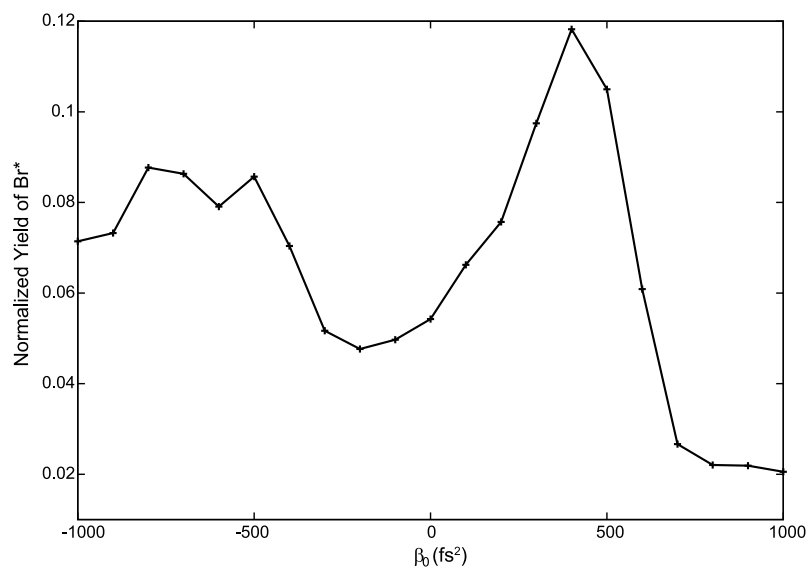

FIG. 6. Same as Fig. 5, except that the time delay between the two pulses now is $1200 \mathrm{fs}$.

in the neighborhood of $1050 \mathrm{fs}$, it is clear that the quadratic spectral chirp is not equivalent to the time delay which can be introduced by a linear spectral phase function. Figure 6 shows the branching ratio as a function of the same linear temporal chirps as in Fig. 5, now at a fixed delay time of $1200 \mathrm{fs}$. The different chirps can lead to an increase as well as a decrease in the branching ratio compared to the unchirped pulse, $\beta_{0}=0$. We also observe a maximum in the branching ratio close to 0.12 , whereas the maximum in Fig. 5 is around 0.1 .

\section{CONCLUSIONS}

To conclude, weak field (one-photon) phase control of photo-fragments is not possible in the long-time limit when the initial state is a single eigenstate but two sequential pulsed one-photon excitations at a fixed time delay lead to the possibility of controlling the fragment states via pure laser-phase manipulation. To that end, we presented a novel form of a theoretical expression, Eq. (4), which suggests that pure (nonlinear) phase modulation can change the quantum mechanical interference terms in the probability amplitudes originating from simultaneous excitation of a sum of different initial (eigen)-states. This expression emphasizes also that the choice of different delay times of the second pulse, in weak-field two-pulse schemes, is just a special case of more general phase modulations of the second pulse.

We considered the photofragmentation dynamics of $\mathrm{IBr}$ leading to $\mathrm{Br}$ in two different electronic states and we have shown that phase modulation of a fixed energy laser pulse can modify the branching ratio between the two electronic states in the photofragmentation process. The suggested implementation for IBr should be experimentally feasible. It would also be interesting to apply this approach of two sequential one-photon excitations to other types of processes, e.g., to the control of competing bond breaking and cis-trans isomerization.

${ }^{1}$ S. A. Rice and M. Zhao, Optical Control of Molecular Dynamics (Wiley, New York, 2000).

${ }^{2} \mathrm{M}$. Shapiro and P. Brumer, Quantum Control of Molecular Processes (Wiley, New York, 2012).

${ }^{3}$ C. Brif, R. Chakrabarti, and H. Rabitz, New J. Phys. 12, 075008 (2010).

${ }^{4}$ A. Assion, T. Baumert, M. Bergt, T. Brixner, B. Kiefer, V. Seyfried, M. Strehle, and G. Gerber, Science 282, 919 (1998).

${ }^{5}$ T. C. Weinacht, J. Ahn, and P. H. Bucksbaum, Nature 397, 233 (1999).

${ }^{6}$ R. J. Levis, G. M. Mekir, and H. Rabitz, Science 292, 709 (2001).

${ }^{7}$ G. Vogt, G. Krampert, P. Niklaus, P. Nuernberger, and G. Gerber, Phys. Rev. Lett. 94, 068305 (2005).

${ }^{8}$ I. Barth and J. Manz, Angew. Chem., Int. Ed. 45, 2962 (2006).

${ }^{9}$ B. J. Sussman, D. Townsend, M. Yu. Ivanov, and A. Stolow, Science 314, 278 (2006).

${ }^{10}$ M. E. Corrales, J. González-Vázquez, G. Balerdi, I. R. Solá, R. de Nalda, and L. Bañares, Nat. Chem. 6, 785 (2014).

${ }^{11}$ N. E. Henriksen, Chem. Phys. 442, 2 (2014).

${ }^{12}$ P. Brumer and M. Shapiro, Chem. Phys. 139, 221 (1989).

${ }^{13}$ M. Shapiro and P. Brumer, J. Phys. Chem. A 105, 2897 (2001).

${ }^{14}$ M. Spanner, C. A. Arango, and P. Brumer, J. Chem. Phys. 133, 151101 (2010).

${ }^{15}$ A. García-Vela and N. E. Henriksen, J. Phys. Chem. Lett. 6, 824 (2015).

${ }^{16}$ C. C. Shu and N. E. Henriksen, J. Chem. Phys. 134, 164308 (2011).

${ }^{17}$ C. C. Shu and N. E. Henriksen, J. Chem. Phys. 136, 044303 (2012).

${ }^{18}$ A. K. Tiwari, D. Dey, and N. E. Henriksen, Phys. Rev. A 89, 023417 (2014).

${ }^{19}$ K. Renziehausen, K. Hader, W. Jakubetz, and V. Engel, ChemPhysChem 14, 1464 (2013).

${ }^{20}$ K. Hader and V. Engel, J. Chem. Phys. 140, 184316 (2014).

${ }^{21}$ P. Brumer and M. Shapiro, Chem. Phys. Lett. 126, 541 (1986).

${ }^{22}$ N. E. Henriksen, Adv. Chem. Phys. 91, 433 (1995).

${ }^{23}$ I. Levy, M. Shapiro, and P. Brumer, J. Chem. Phys. 93, 2493 (1990).

${ }^{24}$ H. Guo, J. Chem. Phys. 99, 1685 (1993).

${ }^{25}$ E. Wrede, S. Laubach, S. Schulenburg, A. Brown, E. R. Wouters, A. J. Orr-Ewing, and M. N. R. Ashfold, J. Chem. Phys. 114, 2629 (2001).

${ }^{26}$ C. C. Marston and G. G. Balint-Kurti, J. Chem. Phys. 91, 3571 (1989).

${ }^{27}$ R. Kosloff, J. Phys. Chem. 92, 2087 (1988).

${ }^{28}$ A. K. Tiwari and N. E. Henriksen, J. Chem. Phys. 141, 204301 (2014). 